\title{
The mathematical model and a block diagram of a synchronous motor compressor unit with a system of automatic control of the excitation
}

\author{
Olimjon Toirov ${ }^{1}$, Tulyagan Kamalov ${ }^{2}$, Utkir Mirkhonov ${ }^{3}$, Sardor Urokov ${ }^{4}$, and Dilnoza Jumaeva ${ }^{5}$ \\ ${ }^{1}$ Tashkent State Technical University, DSc., Prof. Head of the Department of Electrical machine, Tashkent, 100095, Uzbekistan \\ ${ }^{2}$ Scientific and Technical Center of JSC Uzbekenergo, DSc., Prof. Head of the Laboratory, Tashkent, Uzbekistan \\ ${ }^{3}$ Bukhara Engineering Technological Institute, Senior Lecturer, Doctoral Student, Department of Energy Audit, Bukhara, Uzbekistan \\ ${ }^{4}$ Tashkent State Technical University, Doctoral Student, Department of Electrical machine, Tashkent, 100095, Uzbekistan \\ ${ }^{5}$ Institute of General and Inorganic Chemistry of the Academy of Sciences of the Republic of Uzbekistan, Tashkent, 100170, Uzbekistan
}

\begin{abstract}
The general requirements for the automatic excitation control system of synchronous motors of a compressor unit are shown, taking into account its operating modes. A mathematical model is presented, a block diagram of the excitation circuits of a synchronous motor of a compressor unit and an exciter, which characterizes the feedback of the internal properties of this part of the system in dynamics. A block diagram of a closed-loop automatic excitation control system is obtained, taking into account the elasticity of the mechanical part and a hard blow in the compressor gas pipeline. The obtained structural diagrams make it possible to determine the transfer functions of the considered system in terms of control and disturbing influences, the characteristic equation, as well as the dependencies of various frequency characteristics, which make it possible to investigate the dynamic indicators (stability, control quality, etc.) of the system.
\end{abstract}

\section{Introduction}

In large compressor stations, as a rule, synchronous motors (SM) are used as a drive motor for compressors [1-3]. The widespread use of a synchronous motor is due to the fact that they allow the rational use of electrical energy from the mains, have high static and dynamic stability [4-6]. The main advantage of a synchronous motor over asynchronous motors is that by changing the excitation current of the SM, it is possible to change the reactive power. Depending on the excitation current, reactive power can be supplied to the network (during over excitation) and consumed from the network (under over excitation) [7-15].

Excitation systems must satisfy the operation of synchronous machines in normal and emergency conditions. Moreover, they must provide [16-17]:

- the necessary power of the excitation sources;

- the required range of changes in excitation as a function of the parameters of the synchronous machine mode and the range of action of the automatic controller;

- the appropriate limit value of the excitation;

- the certain slew rate of the rotor current of the synchronous motor.

The analysis of various laws of regulation of SM excitation is given in the literatures [16-19].

The analysis of the dynamic modes of systems with SM with automatic control of excitation (ACE) can be carried out on the basis of the method of structural diagrams, which has been widely used in the theory and practice of automatic regulation and control. Representation of SM with automatic regulation of excitation in the form of structural diagrams makes it possible to characterize them from the point of view of some general properties inherent in a certain class of automatic regulation and control systems (structurally stable and unstable systems, multi-loop and multiconnected systems, etc.).

The purpose of the article is to develop a mathematical model and a block diagram of a synchronous motor of a compressor unit with an automatic excitation control system to maintain a constant power factor of the engine with and without taking into account the phenomenon of a hard blow in the compressor gas pipeline.

\section{Research methods}

Consider mathematical models and structural diagrams of excitation control systems of the synchronous motor at constant power factor at nominal frequency network and changing the supply voltage and the load on the shaft. The functional diagram of this system is shown in the Fig. 1 [3].

To increase the efficiency of stabilization of $\cos \varphi$ and simplification of the scheme of ACE, an electric machine system of ACE is proposed, which was developed in the laboratory of "Automated electric drive" of the Institute of Power Engineering and 
Automation of the Academy of Sciences of the Republic of Uzbekistan.

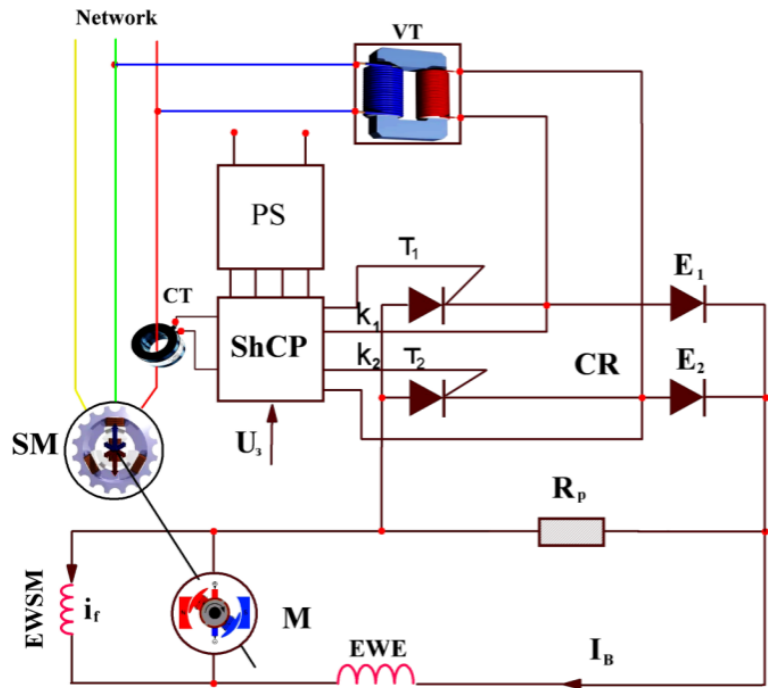

Fig. 1. ACE scheme for the constancy of $\cos \varphi$ : VT - voltage transformer; CT - current transformer; PS - power source, $\mathrm{ShCP}$ - shaper of control pulses; $\mathrm{U}_{3}$ - set point voltage in the IGF; CR - controlled rectifier; Rp - regulatory resistance in the exciter circuit of the exciter; E - exciter; EWE - excitation winding of the exciter; $\mathrm{I} 0$ - rectifier current; Iec - exciter current; if - excitation current of the SM; EWSM - excitation winding of a synchronous motor.

The CR is supplied from the secondary winding of the VT. The output CT winding is connected to the input of the ShCP, from the output of which we obtain control pulses shifted by the set voltage $\mathrm{U}_{3}$ by a certain angle relative to the output voltage of the VT transformer in order to set the set value of the $\cos \varphi \mathrm{SM}$. The CR output is connected in parallel with the regulating resistance and counter-sequentially with the exciter armature and the EWE. The exciter output is connected to the excitation winding of the SM [3].

When analyzing transient processes, we neglect the electromagnetic transient processes in the stator windings of the SM due to their short duration in comparison with mechanical transient processes, and also neglect the active resistance of the stator winding due to its smallness in comparison with the reactance of the SM. We also consider that the magnetic circuits of the SM and the exciter are not saturated. Then the equations of the transient processes of the stator winding are written as the equations of the steady-state mode, and in the equations it is possible to go from the instantaneous values of currents and voltages to their effective values.

If we take into account that the compressor is a mechanism with long operating modes and the load on the drive motor shaft is calm, then the simplifying assumptions made are acceptable.

In this work [16], mathematical models of dynamic processes in the rotating mechanical part of the system of turbo mechanisms and the corresponding one mass and two mass structural schemes were obtained. Also presented are mathematical models and structural diagrams of dynamic processes of electromechanical systems with and without taking into account the phenomenon of hard impact.

\section{Results and discussion}

The electromagnetic torque of the motor is determined by the equation

$$
M=\frac{m K_{e}}{X_{d}} i_{f} U \sin \theta+\frac{m}{2}\left(\frac{1}{X_{q}}-\frac{1}{X_{d}}\right) U^{2} \sin 2 \theta,
$$

where $m$ - number of phases; $K_{e}$ - constant coefficient; $i_{f}$ - field winding current; $U$ - supply voltage; $X_{d}$ and $X_{q}$ synchronous inductive resistances along the longitudinal and transverse axes; $\theta$ - load angle.

Equation (1) for small deviations of the variables takes the form

$$
\Delta M=K_{1 u} \Delta U+K_{1 f} \Delta i_{f}+K_{1 \theta} \Delta \theta,
$$

where

$$
K_{1 u}=a_{1} i_{f 0} \sin \theta_{0}+a_{2} U_{0} \sin 2 \theta_{0},
$$

$K_{1 f}=a_{1} U_{0} \sin \theta_{0} K_{1 \theta}=a_{1} i_{f 0} U_{0} \cos \theta_{0}+a_{2} U_{0}^{2} \cos 2 \theta_{0}$,

$a_{1}=m K_{e} / X_{d}, \quad a_{2}=m\left(X_{d}-X_{q}\right) / X_{d} X_{q}$

The field winding equation has the form

$$
U_{f}=r_{f} i_{f}+L_{f} \frac{d i_{f}}{d t},
$$

or in operator form in increments

$$
\begin{aligned}
& U_{f}=r_{f} i_{f}+L_{f} \frac{d i_{f}}{d t}, \\
& \Delta U_{f}=r_{f} i_{f}\left(T_{f} p+1\right),
\end{aligned}
$$

where $\Delta U_{f}$ - voltage increment of the excitation winding of the synchronous motor, equal to the exciter armature voltage, $T_{f}=L_{f} / r_{f}$ - the time constant of the excitation winding of the synchronous motor, $r_{f}$ and $L_{f}$ - active resistance and inductance of the excitation winding of the synchronous motor.

Equations of electromotive force (EMF) equilibrium in the armature and in the excitation circuit of the exciter will be

$$
U_{f}=E_{a}-r_{a}\left(i_{f}+i_{B}\right)
$$

and

$$
U_{f}=\left(r_{B}+R_{p}\right) i_{B}+L_{B} \frac{d i_{B}}{d t}-R_{p} I_{0},
$$

where $r_{a}$ - active resistance of the armature circuit, $E_{a}-$ armature EMF, $U_{0}=R_{p} I_{0}-$ output voltage of the controlled rectifier. 
The magnetic circuit of the excitation generator is usually unsaturated. Therefore, with a sufficient degree of approximation, we can assume

$$
E_{a}=K_{f l} i_{B} \omega
$$

where $K_{f l}$ - coefficient of proportionality between the flux and the excitation current, $\omega$ - angular velocity of the pathogen.

From (5) and (7) we find

$$
U_{f}=K_{f l} i_{B} \omega-r_{a}\left(i_{f}+i_{B}\right),
$$

Equations (6) and (8) in operator form increments can be written

$$
\Delta i_{B}=\frac{K_{B}}{T_{B} p+1} \Delta U_{f}+\frac{K_{B}}{T_{B} p+1} \Delta U_{0},
$$

and

$$
\Delta U_{f}=K_{a} \Delta \omega+K_{a}^{\prime} \Delta i_{B}-r_{a} \Delta i_{f}
$$

where $T_{B}=L_{B} K_{B}-$ time constant of the exciter excitation winding circuit.

$$
K_{a}=1 /\left(r_{B}+R_{p}\right), r_{B} \text { and } L_{B}-\text { active resistance and }
$$
inductance of the exciter excitation winding, $K_{a}=K_{f l} \omega_{0}, K_{a}^{\prime}=K_{a}-r_{a}$.

On the basis of equations (4), (9) and (10), a block diagram of the circuits of excitation of the SM and the pathogen was constructed, which is given in the figure 2 . Here the feedback characterizes the internal properties of a given part of the system in dynamics. Block diagram of feedback circuits of the system which is given in Fig. 2.

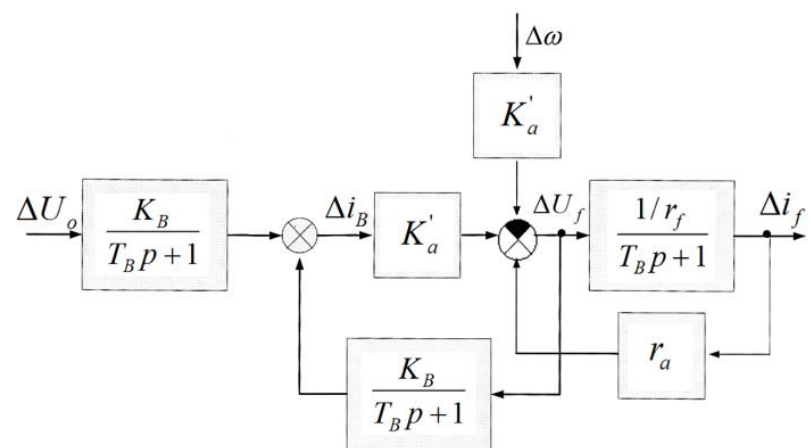

Fig. 2. Block diagram of excitation circuits of the synchronous motor and exciter.

Numerous works are devoted to the study of the dynamic model of a controlled rectifier. In some works, a controlled rectifier is treated as an absolutely noninertial device, in others - as a non-inertial device in a limited frequency band of the control signal, in still others - as a link with a pure delay equal to half the ripple period.
The control characteristic of a single-phase bridge rectifier with an incomplete number of controlled valves is determined by the formula [20]

$$
U_{0}=\frac{\sqrt{6}}{\pi} \frac{U}{K_{T H}}(1+\cos \alpha),
$$

where $U_{0}$ - output voltage of the rectifier, $U$ - phase voltage of the SM, $K_{T H}$ - transformation ratio of the VT, $\alpha$ - rectifier control angle, which is the internal coordinate of the $\mathrm{CR}$.

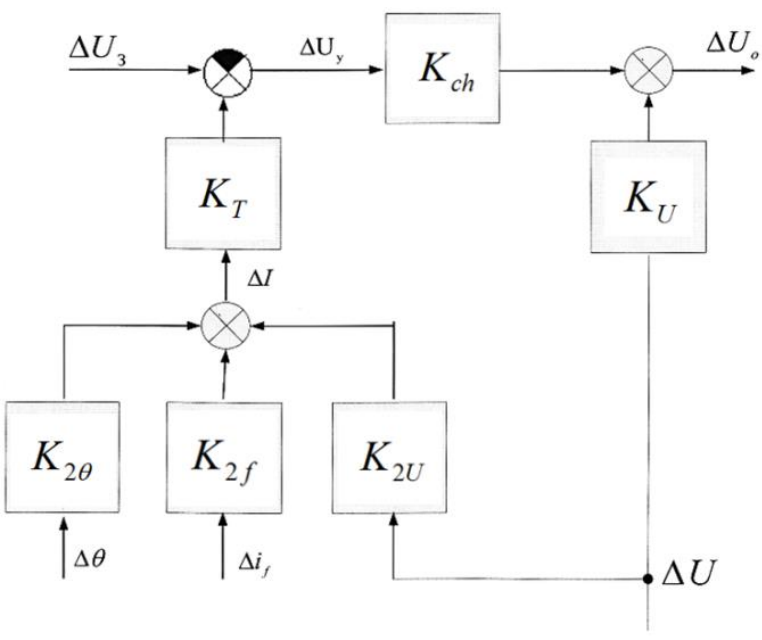

Fig. 3. Block diagram of feedback circuits of the system.

Taking into account small deviations of the equation (11), the following form is obtained

$$
\Delta U_{0}=K_{U} \Delta U+K_{1 \alpha} \Delta \alpha,
$$

where $K_{U}=\frac{\sqrt{6}}{\pi K_{T H}}\left(1+\cos \alpha_{0}\right), K_{U}=\frac{\sqrt{6}}{\pi} \frac{U_{0}}{K_{T H}} \sin \alpha_{0}$

The input coordinate of the $\mathrm{CR}$ is the control voltage $\mathrm{U}$, which in increments is defined as

$$
\Delta U_{y}=\Delta U_{z}-\Delta U_{T}
$$

where $\Delta U_{T}$ - voltage increment across the load of the current transformer.

The voltage across the load of the current transformer depends on the stator current of the synchronous motor

$$
U_{T}=K_{T T} R_{T} I
$$

which in increments will be

$$
\Delta U_{T}=K_{T} \Delta I
$$

where $K_{T}=K_{T T} R_{T}, R_{T}$ and $K_{T T}-$ load resistance and transformation ratio of the current transformer.

Substituting (14) into (13), we obtain

$$
\Delta U_{y}=\Delta U_{z}-K_{T} \Delta I
$$


The expression for the phase current is determined from the vector diagram of the salient-pole SM in the form

$$
I=\frac{1}{X_{d} X_{q}} \sqrt{X_{q}^{2}\left(K_{e} i_{f}-U \cos \theta\right)^{2}+X_{d}^{2} U^{2} \sin ^{2} \theta},
$$

which will be written in increments

$$
\Delta I=K_{2 U} \Delta U+K_{2 f} \Delta i_{f}+K_{2 \theta} \Delta \theta,
$$

where

$$
\begin{aligned}
& K_{2 U}=A\left[X_{d}^{2} U_{0} \sin ^{2} \theta_{0}-\left(K_{e}-U_{0} \cos \theta_{0}\right) X_{q}^{2} \cos \theta_{0}\right], \\
& K_{2 f}=2 K_{e} X_{q}^{2}\left(K_{e} i_{f 0}-U_{0} \cos \theta_{0}\right) A \\
& K_{2 \theta}=A\left[\left(X_{d}-X_{q}\right) U_{0} \cos \theta_{0}-K_{e} i_{f 0} X_{q}^{2}\right] U_{0} \sin \theta_{0}, \\
& A=1 / X_{q} X_{d} \sqrt{X_{q}^{2}\left(K_{e} i_{f 0}-U_{0} \cos \theta_{0}\right)^{2}+X_{d}^{2} U_{d}^{2} \sin \theta_{0}}
\end{aligned}
$$

The control voltage $U_{y}$ is converted through the $\mathrm{ShCP}$ into the control angle of the HC thyristors. Here, the control angle is equal to the phase angle of the secondary voltage of the VT and the current of the CT. The control characteristic is determined by the type of reference voltage. With the sawtooth shape of the reference voltage, the increment of the control angle depends on the increment of the control voltage by dependence [20].

$$
\Delta \alpha=K_{2 \alpha} \Delta U_{y}
$$

where, $K_{2 \alpha}=-\varphi / 2 U_{n m},(\varphi-$ the angular interval of the linear working section of the reference voltage; $U_{n m}-$ maximum value of the reference voltage.

In the general case, depending on the performance of the ShCP system, as an inertial link, the CR is usually reduced to the form of an aperiodic link, a link with a frequent delay, or to both together. However, the inertia of the CR is small, then it makes sense to take it into account only for a low inertial load. For a load with high inertia, it makes no sense to represent a controlled rectifier in the form of an aperiodic link or as a delayed link. From (17) and (12) we obtain

$$
\Delta U=K_{U} \Delta U+K_{c h} \Delta U_{y},
$$

where,

$$
K_{c h}=K_{1 \alpha} K_{2 \alpha}
$$

On the basis of the obtained equations or structural diagrams of individual units and elements of the "SM compressor - gas pipeline" system, structural diagrams of ARV are constructed for constancy of $\cos \varphi$. So, using Fig. 1, 2, 3 and the dependence of the torque increment (2), we obtain a block diagram of the closed-loop ACE system taking into account the elasticity of the mechanical part and the hard shock in the compressor gas pipeline, which is shown in Fig. 4.

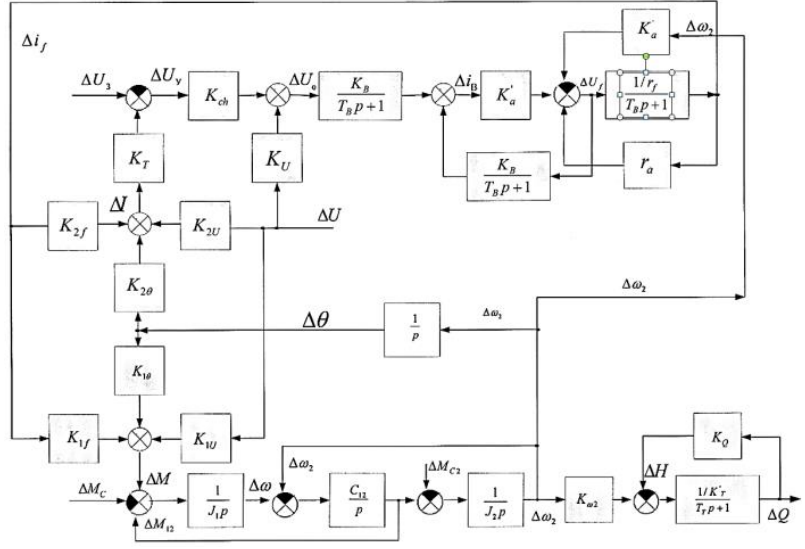

Fig. 4. Block diagram of the system for automatic control of the excitation of the SM taking into account the elasticity of the mechanical part and the hard shock in the compressor gas pipeline.

In Fig. 5 shows a block diagram of systems without taking into account the elasticity of the mechanical part and a hard impact in the gas pipeline, built on the basis of Figs. 2,3,4 and dependence (2). Here the notation is adopted

$$
\begin{gathered}
W_{f}(p)=\frac{\left(T_{B} p+1\right) K_{a}^{\prime} / r_{f}}{\left(T_{f} p-1\right) K_{B} K_{a}^{\prime}-\left(T_{a} p+1\right) r_{a} / r_{f}-\left(T_{f} p+1\right)\left(T_{B} p+1\right)} \\
W_{T}(p)=K_{T}^{\prime} /\left(1+K_{q} K_{T}^{\prime}\right) .
\end{gathered}
$$

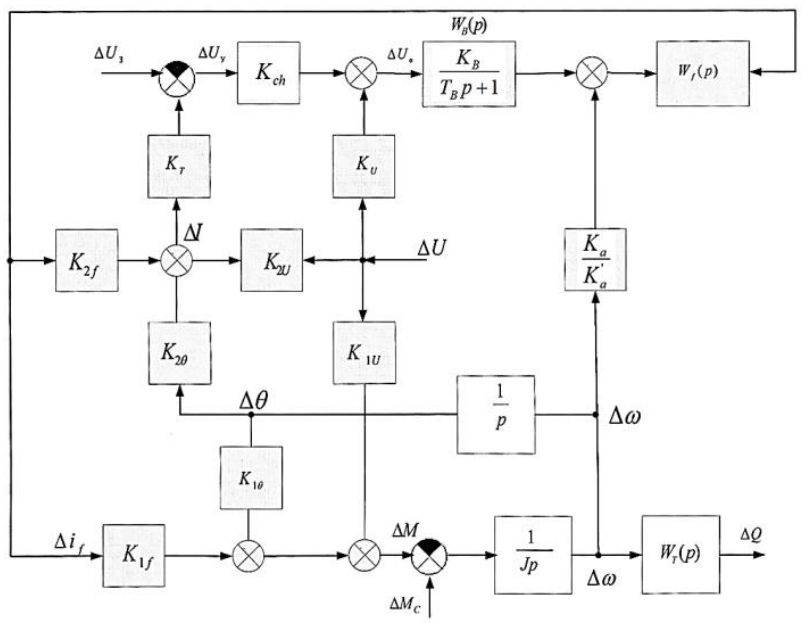

Fig. 5. Block diagram of the system for automatic control of the excitation of the $\mathrm{S} \mid \mathrm{M}$ without taking into account the elasticity of the mechanical part and hard shock in the compressor gas pipeline.

The obtained structural diagrams make it possible to determine the transfer functions of the considered system in terms of control and disturbing influences, the characteristic equation, as well as the dependencies of various frequency characteristics, which make it possible to investigate the dynamic indicators of the system. 


\section{Conclusion}

1. It is shown that it is rational to construct systems for automatic control of excitation of a synchronous electric drive of a compressor unit according to the following principles:

- to maintain the constancy of the set value of the motor power factor for the constancy of the power factor on the buses of the supply substation. When the supply substation does not need fictitious power, the most economical operation of the motor will be with a power factor equal to unity.

- to a minimum of the total losses of electricity in the synchronous motor and the supply network.

2. Linearized mathematical models and structural diagrams of a system for automatic control of excitation of a synchronous motor of a compressor unit for maintaining a constant power factor of the engine with and without taking into account the phenomenon of a hard shock in the compressor gas pipeline are obtained.

3. The obtained structural diagrams make it possible to determine the transfer functions of the considered system in terms of control and perturbing influences, the characteristic equation, as well as the dependences of various frequency characteristics, which make it possible to investigate the dynamic indicators. The system parameters corresponding to this stability region ensure not only stable operation of the system, but also reduce the likelihood of a hard shock phenomenon in the compressor.

\section{References}

1. T.S. Kamalov, Frequency-regulated electric drive of pumping stations of machine irrigation systems, Monograph (Tashkent: Fan, 368, 2014)

2. O. Toirov, U. Mirkhonov, Overview of Compressor Installations and Issues of Their Energy saving, International Journal of Advanced Research in Science Engineering and Technology, 6 (10), 11446-11452 (2019)

3. O. Toirov, U. Mirkhonov, Principles for Controlling the Excitation of Synchronous Motors of the Compressor Installation, International, Journal of Advanced Research in Science, Engineering and Technology, 7 (5), 13876-13881 (2020)

4. O. Toirov, A. Bekishev, S. Urokov, U. Mirkhonov, E3S Web of Conferences, 216, 01116 (2020)

DOI: 10.1051/e3sconf/202021601116

5. O. Toirov, K. Alimkhodjaev, N. Pirmatov, A. Kholbutaeva, E3S Web of Conferences, 216, 01119 (2020) DOI: 10.1051/e3sconf/202021601119

6. H. Afrisal, B. Setiyono, M.F. Yusuf, R.M. Suin, O. Toirov, 7th International Conference on Information Technology, Computer, and Electrical Engineering (ICITACEE), 41-46 (2020)

DOI:

10.1109/ICITACEE50144.2020.9239228
7. A.S. Gusev, S.V. Svechkarev, I.L. Fruitful, An adaptable mathematical model of excitation systems for synchronous machines, Bulletin of the Tomsk Polytechnic University, 308 (7), 211215 (2005)

8. A.Yu. Bukaros, N.O. Romchuk, V.N. Bukaros, Adaptive control system for compressor electric drive, Automation of technological and business processes, 6 (4), 84-90 (2014)

9. S. Tsegaye, K.A. Fante, Analysis of Synchronous Machine Excitation Systems: Comparative Study, Energy and Power Engineering, 10 (12), 14921496 (2018) DOI: 10.5281/zenodo.1130957

10. L. Chen, T. Zheng, S. Mei, X. Xue, B. Liu, Q. Lu, Review and prospect of compressed air energy storage system, Journal of Modern Power Systems and Clean Energy (2016) DOI: 10.1007/s40565-016-0240-5

11. K.P. Timoshchenko, A.A. Yurganov, Key features of the design of control systems for the excitation of synchronous machines, Scientific and technical bulletin of the St. Petersburg State Polytechnic University, 1 (190), 75-81 (2014)

12. T.V. Temgenesvkaya, Methods of tuning automatic regulators excitation of synchronous generators, Modern technologies, System analysis. Modeling, 3 (55), 84-91 (2017)

13. W. Liu, C. Zhu, Modeling and Simulation of Excitation System for Third Harmonic Brushless Synchronous Generator, IOP Conference Series: Materials Science and Engineering (2019) DOI: 10.1088/1757-899X/563/3/032038

14. I.M. Bondar, K.G. Dudarev, V.Yu. Momotov, Voltage regulation of a synchronous generator, Innovative Science, 1, 8-12 (2018)

15. V.I. Voronkov, S.E. Stepanov, V.G. Titov, O.V. Kryukov, Vector control of excitation of synchronous motors of gas-pumping units, Bulletin of the Tula State University, Technical sciences, 3, 204-208 (2010)

16. T.S. Kamalov, K. Muminov, Law of regulation of excitation of synchronous motors of irrigation pumping stations to a minimum of total losses, Uzbek journal Problems of Informatics and Energy, 3-4, 67-71 (1992)

17. D. Bystrov, O. Toirov, G. Mustafakulova, D. Yakubova, NISS2020: Proceedings of the 3rd International Conference on Networking, Information Systems \& Security, 54, 1-3 (2020) DOI: $10.1145 / 3386723.3387873$

18. D. Bystrov, O. Toirov, S. Giyasov, M. Taniev, U. Sardor, NISS2020: Proceedings of the 3rd International Conference on Networking, Information Systems \& Security, 49, 1-4 (2020) DOI: $10.1145 / 3386723.3387868$

19. K. Alimkhodjaev, O. Toirov, M. Taniev, S. Alimkhodjaev, Addressing Issues and Possibility of Introducing Renewable Energy Sources in the Conditions of Uzbekistan, JCR, 7 (15), 17211728 (2020) DOI: $10.31838 /$ jcr.07.15.231

20. V.M. Terekhov, Elements of an automated electric drive (M.: Energoatomizdat, 221, 1987). 